\title{
Stable and Broad Bandwidth Multiwavelength Fiber Ring Laser Incorporating a Highly Nonlinear Photonic Crystal Fiber
}

\author{
Ailing Zhang, Heliang Liu, M. S. Demokan, and H. Y. Tam
}

\begin{abstract}
A stable and broad bandwidth multiwavelength fiber ring laser suitable for use in dense wavelength-division-multiplexing systems is demonstrated. A highly nonlinear photonic crystal fiber is inserted in the ring cavity to stabilize the power fluctuation and broaden the output spectrum. A thin-film Fabry-Pérot etalon filter is used to get stable wavelength lasing. A Sagnac filter is employed to achieve uniform power distribution at each wavelength at the laser output. A laser with a power fluctuation about $0.1 \mathrm{~dB}$ in each wavelength within 20 min and with wavelength shift of less than $0.016 \mathrm{~nm}$ within $10 \mathrm{~min}$ is achieved. A stable 24 -wavelength lasing operation with wavelength spacing of $0.8 \mathrm{~nm}$ within the 3-dB spectral range of 1573-1594 $\mathrm{nm}$ and signal-to-noise ratio better than $48 \mathrm{~dB}$ is observed experimentally.
\end{abstract}

Index Terms-Dense wavelength-division multiplexing (DWDM), erbium-doped fiber, Fabry-Pérot (FP) etalon filter, four-wave mixing (FWM), multiwavelength fiber lasers, nonlinear optics, photonic crystal fiber (PCF).

\section{INTRODUCTION}

$\mathbf{M}$ ULTIWAVELENGTH fiber lasers have aroused interest because of their potential applications in dense wavelength-division-multiplexing (DWDM) systems. The requirements for DWDM sources include a large number of channels over a broad bandwidth, uniformly distributed power over channels, precise and stable wavelength spacing, and small power fluctuation at each channel. These multiwavelength sources can also find applications in fiber-optic sensors and optical testing and measurements. Both the erbium-doped fiber amplifier (EDFA) and the semiconductor optical amplifier (SOA) have been used as the gain medium in developing the multiwavelength fiber laser. The recently developed SOA-based multiwavelength lasers have a typical bandwidth of 20-30 nm and around 0.6-dB peak power fluctuation [1], [2]. EDFA-based multiwavelength lasers often have a smaller bandwidth and worse stability than the SOA-based ones due to their homogenous line broadening characteristics at room temperature.

Several schemes have been proposed to mitigate the mode competition existing within the EDFA at room temperature, which include adding a frequency shifter or phase shifter into the ring cavity [3]-[5] or adding a length of highly nonlinear

Manuscript received July 20, 2005; revised September 12, 2005. This work was supported by the Research Committee of The Hong Kong Polytechnic University (Project A-PG55).

The authors are with the Photonics Research Center and Department of Electrical Engineering, The Hong Kong Polytechnic University, Hung Hom, Hong Kong (e-mail: eealzhan@polyu.edu.hk).

Digital Object Identifier 10.1109/LPT.2005.859542

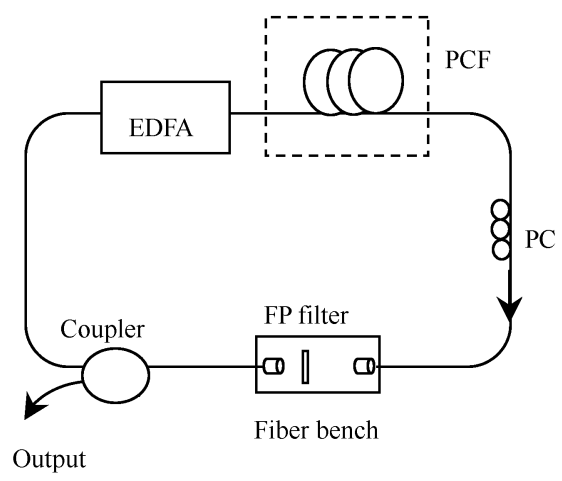

Fig. 1. Schematic of the setup for a multiwavelength fiber laser which utilizes a highly nonlinear PCF and an FP etalon serving as a comb-filter.

photonic crystal fiber (PCF) into the ring cavity [6]. The emergence of PCFs with high nonlinearity and small dispersion slope has enabled the nonlinear effect, such as four-wave mixing (FWM), to happen more easily in a short length of PCF and with low optical power. A dual wavelength fiber laser stabilized by the two degenerate FWM in PCF has been demonstrated [6]. The power fluctuation is greatly reduced by adding a 51-m-long PCF into the ring cavity. However, in [6], only two wavelengths are generated. In order to generate more wavelengths, the two fiber Bragg filters are replaced with a thin-film filter.

In this letter, a stable and broad bandwidth multiwavelength laser source using a highly nonlinear PCF as the nonlinear gain medium is demonstrated. The wavelength spacing of the laser source is anchored on the ITU frequency grid for DWDM application by using a Fabry-Pérot (FP) thin-film filter. The nonlinear effect in the PCF will help broaden the multiwavelength bandwidth and stabilize the power fluctuation at each wavelength. The thin-film filter used has a smaller wavelength drift, which will result in stable lasing wavelength at each channel. The power distribution over each channel can be made to be nearly uniform by a filter. As a result, we get a wavelength- and power-stable, broad bandwidth multiwavelength laser source with wavelength spacing around $0.8 \mathrm{~nm}$ and with uniform power distribution in each wavelength. A detailed explanation follows.

\section{EXPERIMENTAL SETUP}

The schematic of the experimental setup for the multiwavelength fiber ring laser is shown in Fig. 1, which consists of a high-power EDFA, a short length of highly nonlinear PCF, a thin-film FP etalon filter, and a polarization controller (PC). 
The saturation power of the EDFA is about $27 \mathrm{dBm}$, which is used as the gain medium in the ring laser. Isolators are used to provide unidirectional lasing oscillation, which are included inside the EDFA. There is no gain flattening component inside the EDFA. The length of the highly nonlinear PCF is about $20 \mathrm{~m}$, which is used to broaden the spectrum and stabilize the power fluctuation of the ring laser by the FWM effect, as will be explained later. The total insertion loss of the PCF, including the two splices to singlemode fibers, is around $1 \mathrm{~dB}$. The nonlinear coefficient of the PCF is about $30.6 \mathrm{~W}^{-1} \cdot \mathrm{km}^{-1}$, the zero-dispersion wavelength of the PCF is around $1550 \mathrm{~nm}$, and the dispersion slope near the zero-dispersion wavelength is very small $\left[\sim 0.0004 \mathrm{ps} /\left(\mathrm{nm}^{2} \cdot \mathrm{km}\right)\right]$. The stimulated Brillouin scattering threshold is measured to be about $200 \mathrm{~mW}$. The PC is used to control the states of the polarization in the laser cavity. The FP filter is used to provide periodic loss in the spectrum domain to generate multiwavelength lasing. Normally, the maximum handling power of commercial pigtailed thin-film filters is around $20 \mathrm{dBm}$. In our experiment, the power in the cavity is larger than $27 \mathrm{dBm}$. In order to avoid damaging the thin-film filter, a fiber bench from Optics For Research is used to increase the beam diameter to $0.5 \mathrm{~mm}$, which is more than 2.5 times larger than the typical beam diameter of commercial pigtailed devices $(<0.2 \mathrm{~mm})$. So the intensity gets smaller for the same power. As a result, the maximum power allowed to pass through the FP etalon film is increased by more than six times. The loss spectrum of the FP filter including the loss introduced by the fiber bench (around $1.15 \mathrm{~dB}$ ) is observed. The insertion loss at each wavelength peak is around $1.8 \mathrm{~dB}$. The FP filter has an extinction ratio larger than $12 \mathrm{~dB}$. The wavelength spacing of the FP filter is around $0.8 \mathrm{~nm}$. The peak wavelengths and wavelength spacing can be slightly changed by rotating the FP etalon by a small angle.

\section{RESULTS AND DISCUSSION}

First, a 90/10 coupler, where $10 \%$ of the optical power is extracted as output, was used in the laser configuration. The output spectrum of the multiwavelength laser with the maximum biasing current of the pump of the EDFA is shown in Fig. 2(a). The total output power is around $15 \mathrm{dBm}$. The maximum extinction ratio of the multiwavelength laser is about $55 \mathrm{~dB}$. If we define the number of effectively excited wavelengths as those whose powers are within $20 \mathrm{~dB}$ of the power of the strongest wavelength, then the spectrum of the excited wavelengths, from Fig. 2(a), is between 1560 and $1587 \mathrm{~nm}$. The spectrum bandwidth is about $27 \mathrm{~nm}$. The measured 3-dB bandwidth of one channel is about $0.067 \mathrm{~nm}$. The broadened spectrum is caused by the high nonlinearity in the PCF. When several wavelengths are excited in the ring laser, those wavelengths with sufficient power will generate new wavelengths through FWM in the highly nonlinear PCF. The newly generated wavelengths could then generate more wavelengths. The spectrum will become even broader if we increase the value of $\gamma P L$, where $\gamma$ is the nonlinear coefficient of the PCF, $P$ is the power passing through PCF, and $L$ is the effective fiber length of the PCF. In order to increase the optical power $P$ in the laser cavity, the 90/10 coupler is replaced by a 99/1 coupler

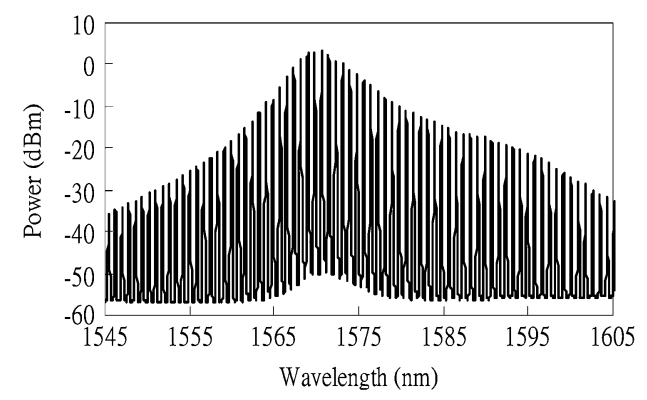

(a)

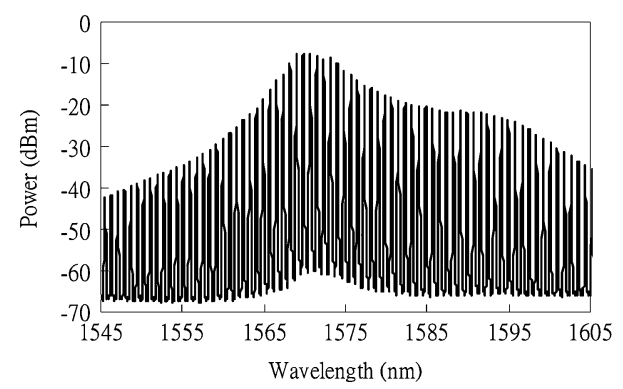

(b)

Fig. 2. Output spectrum of the laser with different couplers.

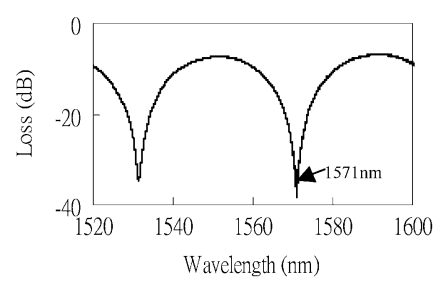

Fig. 3. Sagnac filter spectrum.

to couple $99 \%$ of the output power back to the laser cavity. The output spectrum of the laser becomes wider, as shown in Fig. 2(b). The total output power is around $5.12 \mathrm{dBm}$. The maximum extinction ratio of the multiwavelength laser is still about $55 \mathrm{~dB}$. The spectrum of the excited wavelengths with power drop within 20-dB ranges from 1560 to $1599 \mathrm{~nm}$, as seen in Fig. 2(b). The spectrum range is about $39 \mathrm{~nm}$ which is larger than that obtained by using a 90/10 coupler. The measured $3-\mathrm{dB}$ bandwidth of one channel is about $0.063 \mathrm{~nm}$, which is a little bit smaller than that obtained with a 90/10 coupler. The wavelength spacing is $0.8 \mathrm{~nm}$ for both cases. It is also clear that the output power distribution over wavelengths becomes slightly more flat when a 99/1 coupler is used. However, the channel with the most power is at $1571 \mathrm{~nm}$, irrespective of the coupler used.

In addition to wavelength and power stability, uniform power distribution over each wavelength is also desirable. In order to make the power distribution over different channels nearly uniform, a Sagnac loop filter [7] with a transmission spectrum that is a mirror image of the laser output spectrum is designed and inserted at the laser output to flatten its spectrum [shown in Fig. 2(b)]. The Sagnac loop filter was constructed with a 3-dB coupler, a polarization-maintaining (PM) fiber, and a PC. The Sagnac filter bandwidth can be adjusted by changing the length of the PM fiber. Fig. 3 shows the transmission of the designed 


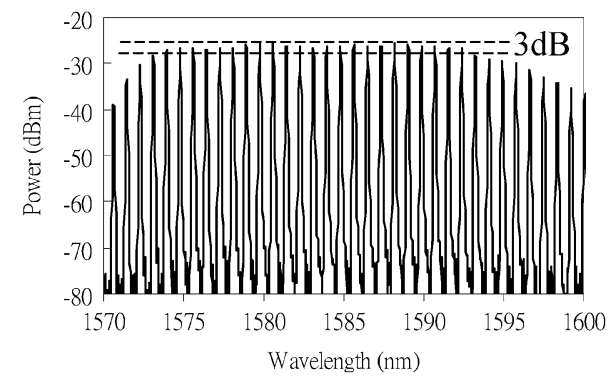

Fig. 4. Output spectrum after filtered by a Sagnac filter.

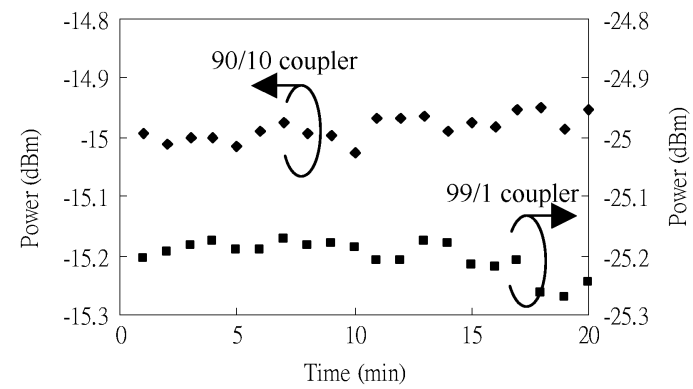

Fig. 5. Power stability of the multiwavelength laser at wavelength $1560.7 \mathrm{~nm}$.

Sagnac loop filter by using a 10-cm PM fiber. The loss peaks can be tuned by changing the polarization state of the PC. In order to flatten the peak at $1571 \mathrm{~nm}$ of the laser output in Fig. 2(b), the loss peak is tuned to $1571 \mathrm{~nm}$ by the PC. The filter is connected at the output of the laser. After the filter the laser spectrum is flattened because the loss of the Sagnac filter at $1571 \mathrm{~nm}$ is much higher than the adjacent wavelengths, whereas the spectrum of the laser is most powerful at $1571 \mathrm{~nm}$ but less so at other wavelengths. Therefore, a balance is established. Fig. 4 focuses on the flat part of the laser spectrum. From Fig. 4, we see that the 3-dB bandwidth of the multiwavelength laser is around $19 \mathrm{~nm}$ with 24 DWDM channels. The extinction ratio of the laser is better than $48 \mathrm{~dB}$. It can be observed from Fig. 4 that the optical power at $1571 \mathrm{~nm}$ is much lower than that at $1585 \mathrm{~nm}$. This is because the extinction ratio of the Sagnac filter is too large. The bandwidth of the multiwavelength laser can be even larger if the filter spectrum is optimized. The uniform power distribution can also be realized by the insertion of a custom-designed filter directly inside the laser cavity.

The output power fluctuations of the laser, using either the 90/10 coupler or the 99/1 coupler, are studied by filtering out the channel at $1560.7 \mathrm{~nm}$ with a bandpass filter. The Sagnac filter is sensitive to the environment which causes its transmission spectrum to drift. In our setup, the Sagnac filter was properly protected to reduce any external disturbances to the filter. In addition, due to the short fiber-length in the Sagnac filter and be- cause the filter has a large bandwidth (free-spectral range), drift in the filter transmission spectrum would introduce only a relatively small variation in the laser output spectrum. In this part of the experiment, the Sagnac filter is not included in the setup. The power fluctuations measured by a power meter are shown in Fig. 5. The power fluctuations for both cases are around $0.1 \mathrm{~dB}$ within $20 \mathrm{~min}$. The wavelength drift of the output laser is also studied by monitoring one wavelength. The wavelength drifts about $0.016 \mathrm{~nm}$ within $10 \mathrm{~min}$, which is much smaller than the channel spacing.

\section{CONCLUSION}

A stable and broad bandwidth multiwavelength fiber ring laser for use in DWDM systems has been demonstrated. The output spectrum is broadened and power fluctuation is stabilized by means of the nonlinear effect in a highly nonlinear PCF. Stable wavelengths are achieved by using a thin-film FP etalon filter. The power distribution over channels is made nearly uniform by using a Sagnac filter at the output of the laser such that the 3-dB bandwidth of the laser is around $19 \mathrm{~nm}$ with 24 -wavelengths. The wavelength drift is less than $0.016 \mathrm{~nm}$ within $10 \mathrm{~min}$ and the power fluctuation in one wavelength is about $0.1 \mathrm{~dB}$ within $20 \mathrm{~min}$.

\section{ACKNOWLEDGMENT}

The authors wish to thank P. K. A. Wai for fruitful discussions.

\section{REFERENCES}

[1] H. Dong, G. Zhu, Q. Wang, H. Sun, N. K. Dutta, J. Jaques, and A. B. Piccirilli, "Multiwavelength fiber ring laser source based on a delayed interferometer," IEEE Photon. Technol. Lett., vol. 17, no. 2, pp. 303-305, Feb. 2005.

[2] Y.-G. Han, G. Kim, J. H. Lee, S. H. Kim, and S. B. Lee, "Lasing wavelength and spacing switchable multiwavelength fiber laser from 1510 to 1620 nm," IEEE Photon. Technol. Lett., vol. 17, no. 5, pp.989-991, May 2005.

[3] F. Ahmed, N. Kishi, and T. Miki, "Multiwavelength Erbium-doped fiber Fabry-Pérot laser and its uniform spectral lines power operation," IEEE Photon. Technol. Lett., vol. 17, no. 4, pp. 753-755, Apr. 2005.

[4] J. Yao, J. Yao, Z. Deng, and J. Liu, "Multiwavelength Erbium-doped fiber ring laser incorporating and SOA-based phase modulator," IEEE Photon. Technol. Lett., vol. 17, no. 4, pp. 756-758, Apr. 2005.

[5] A. Bellemare, M. Karasek, M. Rochette, S. LaRochello, and M. Tetu, "Room temperature multifrequency erbium-doped fiber lasers anchored on the ITU frequency grid," J. Lightw. Technol., vol. 18, no. 6, pp. 825-831, Jun. 2000.

[6] X. Liu, X. Yang, F. Lu, J. Ng, X. Zhou, and C. Lu, "Stable and uniform dual-wavelength erbium-doped fiber laser based on fiber Bragg gratings and photonic crystal fiber," Opt. Express, vol. 13, no. 1, pp. 142-147, Jan. 2005.

[7] X. Fang and R. O. Claus, "Polarization-independent all-fiber wavelength-division multiplexer based on a Sagnac interferometer," Opt. Lett., vol. 20, no. 20, pp. 2146-2148, Oct. 1995. 